\title{
Integral field optical spectroscopy of a representative sample of ULIRGs
}

\section{Two-dimensional kpc-scale extinction structure ${ }^{\star}$}

\author{
M. García-Marín ${ }^{1,2}$, L. Colina ${ }^{1}$, and S. Arribas ${ }^{1}$ \\ 1 Departamento de Astrofísica Molecular e Infrarroja, Instituto de Estructura de la Materia, CSIC C/ Serrano 121, Madrid, Spain \\ e-mail: maca@ph1.uni-koeln.de \\ 2 Now at the I. Physikalisches Institut, Universität zu Köln, Zülpicher Strasse 77, 50937 Köln, Germany
}

Received 20 May 2009 / Accepted 11 June 2009

\section{ABSTRACT}

\begin{abstract}
Context. Ultraluminous infrared galaxies are merging systems characterized for containing large amounts of dust and emitting the bulk of their energy in the infrared. Dust affects several of the observed properties derived from optical and near-IR rest-frame data, such as the stellar morphology and the ionized gas distribution. Systematic analysis of the dust distribution in representative samples of ULIRGs are needed to investigate its two-dimensional structure, and to establish its impact in the derivation of fundamental properties such as star formation rates, effective radii, and dynamical masses.

Aims. We investigate the two-dimensional kpc-scale structure of the extinction in a representative sample of local ULIRGs using the $\mathrm{H} \alpha / \mathrm{H} \beta$ line ratio.

Methods. We use optical integral field spectroscopy obtained with the INTEGRAL instrument at the William Herschel Telescope. Complementary optical and near-IR high angular resolution HST images have also been used. Compared to classical optical long-slit analysis, our data provides information about the entire system, and not only in pre-selected orientations.

Results. The extinction exhibits a very complex and patchy structure in ULIRGs on kpc scales, from basically transparent regions to others deeply embedded in dust $\left(A_{\mathrm{V}} \simeq 0.0\right.$ to $\left.A_{\mathrm{V}} \simeq 8.0 \mathrm{mag}\right)$. Nuclear extinction covers a broad range in $A_{\mathrm{V}}$ from 0.4 to $7.7 \mathrm{mag}, 69 \%$ of the nuclei having $A_{\mathrm{V}} \geq 2.0 \mathrm{mag}$. Extinction in the external regions is substantially lower than in the nuclei with $60 \%$ of the ULIRGs in the sample having median $A_{\mathrm{V}}$ of less than 2 mag for the entire galaxy. While post-coalescence nuclei tend to cluster around $A_{\mathrm{V}}$ values of 2 to $3 \mathrm{mag}$, pre-coalescence nuclei appear more homogeneously distributed over the entire $0.4 \mathrm{mag} \leq A_{\mathrm{V}} \leq 7.7 \mathrm{mag}$ range. For the average extinction $\left(A_{\mathrm{V}} \simeq 2.0\right)$ derived for the ULIRGs of the sample, the ratio of the de-reddened to observed $S F R$ values is 6. The extinction-corrected, $\mathrm{H} \alpha$-based $S F R$ ranges from 10 to $300 M_{\odot} \mathrm{yr}^{-1}$. For only $27 \%$ of the cases the de-reddened $S F R$ is $<20 M_{\odot} \mathrm{yr}^{-1}$, whereas for the observed $S F R$ this percentage increases to 87\%. The IR-based SFR is always higher than the opticalbased one, with differences ranging from about 2 to up to 30. The nuclear observed $S F R$ has an average contribution to the total one of $16 \%$ for the entire sample. Once corrected for extinction, the average value becomes $31 \%$. Because of mostly extinction effects, the optical ( $I$-band) half-light radius in the sample galaxies is on average a factor 2.3 larger than the corresponding near-IR $(H$-band) value.
\end{abstract}

Key words. galaxies: interactions - galaxies: structure - galaxies: fundamental parameters

\section{Introduction}

The significance of galaxies with an infrared excess was highlighted early by Low \& Kleinmann (1968) and Rieke \& Low (1972), and lately recognized by observations of the IRAS satellite (Soifer et al. 1984). Among the sources that emit the bulk of their energy in the infrared, the Ultra-Luminous Infrared Galaxies (ULIRGs, $10^{12} L_{\odot} \leq L_{\text {bol }} \sim L_{\mathrm{IR}}[8-1000 \mu \mathrm{m}] \leq$ $10^{13} L_{\odot}$ ), are amongst the brightest objects in the local Universe (see reviews in Lonsdale et al. 2006; Sanders \& Mirabel 1996). The process of merging with accompanying starbursts plays a key role in these galaxies, and appears to transform spirals

\footnotetext{
* Based on observations with the William Herschel Telescope operated on the island of La Palma by the ING in the Spanish Observatorio del Roque de los Muchachos of the Instituto de Astrofísica de Canarias. Based also on observations with the NASA-ESA Hubble Space Telescope, obtained at the Space Telescope and Science Institute, which is operated by the Association of Universities for Research in Astronomy, Inc. under NASA contract number NAS5-26555.
}

into low-intermediate mass ellipticals (e.g., Colina et al. 2001; Genzel et al. 2001; Tacconi et al. 2002; Naab et al. 2006 and references therein). This star formation activity is the main energy source in ULIRGs, although in some cases the contribution of an obscured AGN is relevant (see Nardini et al. 2008; Risaliti et al. 2006). These galaxies contain large quantities of gas and dust that, independent of the nature of the energy source, are heated re-emitting most of their energy in the infrared.

High angular resolution Hubble Space Telescope (HST) optical and near-infrared images (Farrah et al. 2001; Bushouse et al. 2002) infer that ULIRGs have in general a complex stellar structure. On the one hand, these structures are caused by the merger itself that transforms the stellar distribution in the parent galaxies (i.e., into tidal tails, nuclear and extended starforming regions, and double nuclei) as they evolve through the different phases of the interaction. On the other hand, large amounts of gas and dust settle into extended lanes and/or filaments creating additional structures due to non-uniform extinction effects (see $V, I$, and $H$ band images in Farrah et al. 2001; 
Bushouse et al. 2002). The impact of the dust effects is lower in the near-IR, allowing us to identify the true stellar morphology of the galaxies (e.g., HST observations from Scoville et al. 2000; Bushouse et al. 2002). This is emphasized by studies based on HST color maps (F814W-F606W), which detect a non uniform color structure with blue and red compact knots that are most likely caused by unobscured star formation, and dustenshrouded star formation or remnant nuclei from the progenitor galaxies (Surace et al. 1998; Farrah et al. 2001). Similar results have been obtained for luminous infrared galaxies (LIRGs, $\left.10^{11} L_{\odot} \leq L_{\mathrm{bol}} \sim L_{\mathrm{IR}}[8-1000 \mu \mathrm{m}] \leq 10^{12} L_{\odot}\right)$ using near-IR $H S T$ images. Complex dust features in their centers, with an average optical extinction $\left(A_{\mathrm{V}}\right)$ of between 3 and $5 \mathrm{mag}$, are also observed (Alonso-Herrero et al. 2006).

All the afore mentioned HST imaging studies indicate that although the dust tends to be concentrated in the inner few $\mathrm{kpc}$, the global distribution is very patchy on scales of hundreds to thousands of parsecs, and therefore non-uniform extinction effects play a major role. ULIRGs have been intensively studied with narrow, long-slit spectroscopy (e.g., Veilleux et al. 1995), but despite its importance, the spectroscopic derivation of the two-dimensional dust and gas distribution has not been the subject of much scrutiny. Previous integral field spectroscopy (IFS) studies of specific targets demonstrated the lack of coincidence between the true, dynamical nucleus of the galaxy and the region identified as the optical nucleus, which can be separated by distances from 0.5 (Arp 299, García-Marín et al. 2006) to $1.3 \mathrm{kpc}$ (IRAS 17208-0014, Arribas \& Colina 2003). Other studies have also shown the large differences (of up to a factor of 10) in extinction between the nuclear, circum-nuclear, and extranuclear regions due to the patchy and non-uniform distribution of the dust on kpc-scales (e.g. IRAS 12112-0305, Colina et al. 2000; Arp 299, García-Marín et al. 2006). These results emphasize the importance of knowing (and correcting for) the two-dimensional structure of the extinction before using rest-frame optical lines (mainly $\mathrm{H} \alpha$ ) or structural parameters (e.g., effective radius) in the derivation of star formation rates and dynamical masses in these galaxies, and their intermediate- and high- $z$ analogs.

This is the second paper in a series aimed at studying in detail the internal structure and kinematics of local ULIRGs (García-Marín et al. 2009, Paper I). Here, we present the first systematic two-dimensional analysis of the dust extinction in a representative sample of low $z$. ULIRGs using optical integral field spectroscopy complemented with existing archival $H S T$ high angular resolution images. The paper is organized as follows. The galaxy sample is presented in Sect. 2, whereas Sect. 3 is dedicated to the observations and data reduction, and Sect. 4 presents the data analysis. Section 5 discusses the extinction structure of ULIRGs on kpc scales, and the implications for the derivation of star formation rates and dynamical masses in lowand high- $z$ ULIRGs. Finally, in Sect. 6, a brief summary of the main results is given.

The current analysis that we are carrying out in this sample of ULIRGs is part of a larger survey that is investigating the two-dimensional extinction, ionization, and kinematic kpcscale structure of a representative sample of low- $z$ LIRGs and ULIRGs, and its implications for their high-redshift analogs. The survey is based on the use of optical IFS data obtained with different facilities. The study of northern hemisphere ULIRGs has been performed mostly with the integral field unit (IFU) INTEGRAL (Arribas et al. 1998). Several papers presenting the results for individual (Colina et al. 1999; García-Marín et al. 2006) or small subsamples of galaxies (see Colina et al. 2005;
Monreal-Ibero et al. 2007 and references therein) have already been published.

To include southern systems and extend the sample to lower luminosity objects covering most of the (U)LIRGs range $\left(\log \left(L_{\mathrm{IR}} / L_{\odot}\right)=11.0-12.6\right)$, we observed an additional sample of about 40 systems using VIMOS IFU (LeFevre et al. 2003). The VIMOS sample description and first results were presented in Arribas et al. (2008).

We also observed the majority of the northern hemisphere galaxies from the volume-limited (distances of about 35-75 Mpc) local sample of LIRGs presented in Alonso-Herrero et al. (2006); further details of the selection criteria can be found in Alonso-Herrero et al. (2006). These galaxies, with an average luminosity of $\log \left(L_{\mathrm{IR}} / L \odot\right)=11.32$, were observed with the IFU PMAS (Roth et al. 2005). The data and first results are presented in Alonso-Herrero et al. (2009, Paper I.).

In addition to this, near-IR IFS with SINFONI of a subsample of LIRGs and ULIRGs is also being conducted (see Bedregal et al. 2009 for first results) to investigate the multiwavelength (optical and near-IR) properties of these galaxies.

Throughout this paper, we use $\Omega_{\Lambda}=0.7, \Omega_{M}=0.3$, and $H_{0}=70 \mathrm{~km} \mathrm{~s}^{-1} \mathrm{Mpc}^{-1}$.

\section{The sample of ULIRGs}

Our original sample of ULIRGs with available optical INTEGRAL IFS consists of 22 systems selected to be representative of this galaxy class (details on the sample selection can be found in García-Marín et al. 2009, Paper I). These systems exhibit a large variety of morphologies, from wide pairs to close pairs with well developed tidal tails to single nucleus mergers. The sample covers the luminosity range $11.8 \leq \log \left(L_{\mathrm{IR}} / L_{\odot}\right) \leq$ 12.6, and includes all the different classes of nuclear activity (H II-, LINER-, and Seyfert-like). A few galaxies of the sample are not present in the IFS-based analysis of the dust distribution for a variety of reasons, such as the lack of detection or non coverage of $\mathrm{H} \beta$ (Arp 220 and IRAS 09427+1929), low S/N, which precludes the detection of emission lines (IRAS 13469+5833), limited spatial resolution (IRAS 13342+3932), and severe AGN contamination (Mrk 231). Using the projected distance between the nuclei of the parent galaxies, the sample has been further divided into two categories, pre- (nuclear distance $>1.5 \mathrm{kpc}$ ) and post-coalescence (nuclear distance $\leq 1.5 \mathrm{kpc}$ ). This separation allows us to investigate and discriminate the characteristics of galaxies in the final phases of the merger from those in earlier dynamical stages. Given this, the two-dimensional dust distribution has been derived for 17 (U)LIRGs, nine of them precoalescence systems and eight post-coalescence (Table 1).

\section{Observations and data reduction}

IFS data of the galaxy sample was obtained between 1998 and 2004 using INTEGRAL, a fiber-based optical integral field system (Arribas et al. 1998) connected to the Wide Field Fibre Optic Spectrograph (WYFFOS; Bingham et al. 1994) and mounted on the $4.2 \mathrm{~m}$ William Herschel Telescope. Depending on the structure and compactness of the ULIRGs, three different INTEGRAL configurations were used: the so-called standard bundles 1, 2, and 3 (SB1 with fiber diameter 0.45 arcsec and field-of-view $(\mathrm{FoV}) 7.8 \times 6.4 \operatorname{arcsec}^{2}, \mathrm{SB} 2$ with fiber diameter $0.9 \operatorname{arcsec}$ and FoV $16.0 \times 12.3 \mathrm{arcsec}^{2}$, and SB3 with fiber diameter 2.7 arcsec and FoV $33.6 \times 29.4 \operatorname{arcsec}^{2}$ ). The spectra were taken with a 600 lines $\mathrm{mm}^{-1}$ grating, providing an effective spectral resolution $(F W H M)$ of approximately 6.0, 6.0 and $9.8 \AA$ for the 
Table 1. Extinction and structural properties for the sample of galaxies.

\begin{tabular}{|c|c|c|c|c|c|c|c|c|c|c|c|}
\hline Galaxy $^{a}$ & $\begin{array}{c}\log \left(L_{\mathrm{IR}}\right)^{b} \\
L_{\odot}\end{array}$ & $z$ & $\begin{array}{c}A_{\text {Vmedian }}{ }^{c} \\
\text { (mag) }\end{array}$ & $\begin{array}{l}A_{\mathrm{Vnuc}}{ }^{d} \\
\text { (mag) }\end{array}$ & $\begin{array}{l}A_{\mathrm{Vmax}} e \\
(\mathrm{mag})\end{array}$ & $\begin{array}{l}\operatorname{Sep}^{f} \\
(\mathrm{kpc})\end{array}$ & $\begin{array}{c}\text { Scale } \\
(\mathrm{kpc} / \mathrm{arcsec})\end{array}$ & $\begin{array}{l}R_{\text {eff }}(\text { optical }) \\
\quad(\mathrm{kpc})\end{array}$ & $\begin{array}{l}R_{\mathrm{eff}^{g}} \\
(\mathrm{kpc})\end{array}$ & $\begin{array}{l}R_{\mathrm{eff}^{h}} \\
(\mathrm{kpc})\end{array}$ & Morphology $^{i}$ \\
\hline IRAS $13156+0435 \mathrm{~N}$ & 12.13 & 0.113 & $2.5 \pm 0.8$ & 3.3 & 3.7 & $3.9 \pm 1.0$ & 2.058 & & 5.9 & & IP at $36.0 \mathrm{kpc}$ \\
\hline IRAS $13156+0435 S$ & & & $0.9 \pm 0.4$ & 1.5 & 1.7 & $2.2 \pm 1.0$ & 2.058 & & 4.9 & & \\
\hline RAS $18580+6527 E$ & 12.26 & 0.176 & $1.3 \pm 0.8$ & 0.5 & 3.9 & $20.3 \pm 1.5$ & 2.986 & $11.3^{j}$ & 6.5 & & IP at $15.0 \mathrm{kpc}$ \\
\hline IRAS $18580+6527 \mathrm{~W}$ & & & & 0.8 & 3.9 & $8.7 \pm 1.5$ & 2.986 & $9.4^{j}$ & 4.3 & & \\
\hline RAS $16007+3743 E$ & 12.11 & 0.185 & $1.9 \pm 1.0$ & 4.5 & 5.0 & $12.7 \pm 1.5$ & 3.100 & & 4.0 & & IP at $14.2 \mathrm{kpc}$ \\
\hline IRAS $16007+3743 \mathrm{~W}$ & & & & 4.7 & 5.0 & $1.4 \pm 1.5$ & 3.100 & & 4.2 & & \\
\hline RAS $06268+3509 \mathrm{~N}$ & 12.51 & 0.169 & $2.0 \pm 1.2$ & 5.7 & 6.9 & $3.2 \pm 1.4$ & 2.895 & 8.3 & 2.0 & 2.1 & IP at $9.1 \mathrm{kpc}$ \\
\hline [RAS 06268+3509S & & & & 2.2 & 6.9 & $10.5 \pm 1.4$ & 2.895 & 6.3 & 1.5 & 1.1 & \\
\hline RAS $08572+3915 \mathrm{~N}$ & 12.17 & 0.058 & $1.7 \pm 1.1$ & 2.3 & 5.8 & $0.0 \pm 0.6$ & 1.130 & $2.9^{k}$ & 2.8 & 0.6 & IP at $6.1 \mathrm{kpc}$ \\
\hline [RAS $08572+3915 S$ & & & & 0.4 & 5.8 & $1.3 \pm 0.6$ & 1.130 & 4.3 & 1.4 & 0.6 & \\
\hline $348-1447 \mathrm{~N}$ & 12.39 & 0.083 & $2.9 \pm 1.0$ & 5.4 & 5.8 & $5.6 \pm 0.8$ & 1.556 & $5.3^{l}$ & 1.4 & 1.5 & IP at $5.5 \mathrm{kpc}$ \\
\hline RAs & & & & 4.1 & 5. & $8 \pm$ & 1.556 & $4.1^{l}$ & 3.0 & 1.3 & \\
\hline 690 & 11.81 & 0.010 & $1.9 \pm 0.6$ & 3.7 & 4. & $0.5 \pm 0.1$ & 0.205 & & 1.2 & 0.9 & IP at $5.0 \mathrm{kpc}$ \\
\hline $\operatorname{Arp} 2$ & & & $2.0 \pm 0.6$ & 2.7 & 3.4 & $0.3 \pm 0.1$ & 0.205 & & 2.0 & 0.4 & \\
\hline $2+0305 \mathrm{~N}$ & 12.37 & 0.073 & $1.7 \pm 1.3$ & 3.0 & 7. & $5.5 \pm 0.7$ & 1.395 & 3.3 & 2.0 & 1.7 & IP at $4.0 \mathrm{kpc}$ \\
\hline $2+0305 \mathrm{~S}$ & & & & 7.7 & 7 & $0.0 \pm$ & 1.395 & $4.5^{l}-x-2 \cdot n$ & 1.5 & 1.1 & \\
\hline Mrk 4 & 11.81 & 0.050 & $0.9 \pm 0.5$ & 0.7 & 3. & $4.7 \pm 0.5$ & 0.984 & & 1.0 & 0.2 & $\mathrm{DN}$ at $3.8 \mathrm{kpc}$ \\
\hline $\mathrm{Mrl}$ & & & & 1.5 & 3. & .5 & 0.984 & & 1.0 & 1.0 & \\
\hline & 12.57 & 0.144 & $2.2 \pm 0.6$ & 2.4 & 3 & .6 & 2.522 & & 1.5 & & $\mathrm{DN}$ at $1.5 \mathrm{kpc}$ \\
\hline $7+5351$ & 12.13 & 0.143 & $1.2 \pm 1.0$ & 3.0 & 3.0 & $0.0 \pm 0.6$ & 2.507 & & 8.9 & & $\mathrm{DN}$ at $1.5 \mathrm{kpc}$ \\
\hline Mrk 27 & 12.18 & 0.038 & $1.9 \pm 1.0$ & 3.1 & 4.9 & $2.1 \pm 0.4$ & 0.749 & $4.3^{l}$ & 4.4 & 2.0 & DN at $0.7 \mathrm{kpc}$ \\
\hline RAS & 12.43 & 0.043 & $5.5 \pm 2.6$ & 5.0 & 9.0 & $0.8 \pm 0.4$ & 0.844 & $3.6^{l}$ & 3.2 & 1.5 & SN \\
\hline $0+3609$ & 12.09 & 0.055 & $2.3 \pm 1.7$ & 2.5 & 7.9 & $2.4 \pm 0.5$ & 1.072 & $2.2^{l}$ & 1.9 & 1.4 & SN \\
\hline IRA & 12.07 & 0.101 & $1.3 \pm 0.7$ & 1.1 & 3.3 & $3.6 \pm 0.9$ & 1.854 & & 7.6 & & SN \\
\hline $60+2919$ & 12.18 & 0.117 & $1.5 \pm 0.5$ & 2.2 & 2.5 & $2.3 \pm 1.0$ & 2.113 & & 4.8 & & SN \\
\hline IRAS $15206+3342$ & 12.27 & 0.124 & $0.8 \pm 0.5$ & 1.0 & 2.0 & $2.4 \pm 1.0$ & 2.232 & $2.2^{k}$ & 2.9 & & SN \\
\hline
\end{tabular}

${ }^{a}$ The galaxies are listed according to decreasing nuclear separation (see García-Marín et al. 2009); ${ }^{b} L_{\mathrm{IR}}(8-1000 \mu \mathrm{m}$ ) was derived following Sanders \& Mirabel 1996); ${ }^{c}$ median extinction of the entire system with the deviation that effectively represents the distribution of the extinction values over the entire area where the $\mathrm{H} \alpha$ and $\mathrm{H} \beta$ measurements can be obtained. For pre-coalescence systems with one pointing per nuclei, the median has been calculated for each pointing; ${ }^{d}$ nuclear extinction of the galaxy. The uncertainties are about $20 \% ;{ }^{e}$ maximum extinction of the system; ${ }^{f}$ separation between the optical nucleus, identified as the peak position in the $F 814 \mathrm{~W}$ band of the HST images, and the position of the peak of extinction; ${ }^{g}$ effective radius measured from the HST WFPC2 camera, with the filter $F 814 W$, corresponding to a central wavelength of $0.82 \mu \mathrm{m}$ and a filter width of $0.17 \mu \mathrm{m} ;{ }^{h}$ effective radius measured from the HST NICMOS2 camera, with the filter $F 160 W$, corresponding to a central wavelength of $1.55 \mu \mathrm{m}$ and a filter width of $0.40 \mu \mathrm{m} ;{ }^{i}$ IP means interacting pair, DN double nucleus and SN single nucleus; ${ }^{j}$ effective radius measured from the HST WFPC 2 camera, with the filter $F 606 \mathrm{~W}$, corresponding to a central wavelength of $0.58 \mu \mathrm{m}$, and a filter width of $0.16 \mu \mathrm{m} ;{ }^{k}$ effective radius measured from the HST WFPC2 camera, with the filter $F 439 \mathrm{~W}$, corresponding to a central wavelength of $0.43 \mu \mathrm{m}$ and a filter width of $0.05 \mu \mathrm{m} ;{ }^{l}$ effective radius measured from the HST ACS camera, with the filter $F 435 \mathrm{~W}$, corresponding to a central wavelength of $0.43 \mu \mathrm{m}$ and a filter width of $0.14 \mu \mathrm{m}$.

SB1, SB2, and SB3 bundles, respectively ${ }^{1}$. The covered spectral range of interest was $\lambda \lambda 4500-7000 \AA$ rest-frame. The reduction and calibration of the IFS data were performed inside the IRAF environment, and followed the standard procedures applied to this type of data (see Arribas et al. 1997 and references therein). Full details of the observations and data reduction can be found in García-Marín et al. (2009).

Complementary HST archive images were also used. Specifically, we used optical WFPC2 F439W, F606W, and F814W data, available for 12, 12, and $100 \%$ of the ULIRGs under study, and near-IR NICMOS F160W data, available for $60 \%$ of the sample. Filters $F 439 W, F 814 W$, and $F 160 W$, are the HST analogs to the ground-based Johnson-Cousins $B, I$, and $H$, respectively (Origlia \& Leitherer 2000). The filter $F 606 W$

\footnotetext{
1 These values correspond to the old camera mounted on WYFFOS, that was used for the present observations. From August 2004 a new camera was commissioned for the instrument. See more details in http://www.iac.es/proyecto/integral

2 The IRAF software is distributed by the National Optical Astronomy Observatory (NOAO), which is operated by the Association of Universities for Research in Astronomy (AURA), Inc., in cooperation with the National Science Foundation.
}

is equivalent to a wide $V$-filter. In addition, F435W ACS images, equivalent to the Johnson- $B$ filter and available for $29 \%$ of the sample, were also used. The HST images were calibrated on the fly, with the best available reference files. In the case of NICMOS data, the combination of the dithered individual exposures was repeated. All images were sky (or background) subtracted.

\section{Data analysis}

In all galaxies with available information, the two-dimensional structure of the extinction/dust was derived using the $\mathrm{H} \alpha / \mathrm{H} \beta$ line ratio (Balmer decrement), a foreground dust screen model and a mean interstellar extinction law based on Savage \& Mathis (1979). The line fluxes were obtained by fitting each emission line to a single Gaussian function using the DIPSO package (Howarth \& Murray 1988) inside the STARLINK environment ${ }^{3}$. The presence of underlying stellar hydrogen absorption lines was not detected and therefore no correction was applied when measuring the flux of the corresponding emission lines. This

\footnotetext{
${ }^{3}$ See http://www.starlink.rl.ac.uk/
} 
would introduce a small overestimate in the values derived for the optical extinction since the equivalent widths of the hydrogen emission lines are larger than $40 \AA$ in the extended high surface brightness regions where they are detected, much larger than the $E W_{\text {abs }}(\mathrm{H} \alpha)=E W_{\text {abs }}(\mathrm{H} \beta) \sim 2 \AA$ of the underlying stars.

It is known that different emission line ratios infer different extinction values, indicating that the observed recombination lines originate at different depths in these dusty regions. For this reason, especially in dust-enshrouded environments such as the central regions of ULIRGs where large quantities of dust are detected, the Balmer decrement provides only a lower limit to the true extinction. A clear example of this is the nucleus of the galaxy IC 694, a member of the pre-coalescence system Arp 299. The nuclear extinction derived using the Balmer decrement is $A_{\mathrm{V}} \simeq 3.0$, whereas with the $\mathrm{Pa} \alpha / \mathrm{H} \alpha$ line ratio it is $A_{\mathrm{V}} \simeq 6.0$ (García-Marín et al. 2006).

The HST ACS, WFPC2, and NICMOS images were used to obtain the optical ( $B$ or $V$ and $I$ bands) and near-IR ( $H$ band) effective radius for each of the sample galaxies, defined as the radius that encloses half the luminosity of the galaxy. It was derived using sky- or background-corrected images, using apertures of increasingly large radius being centered on the optical nucleus of each galaxy. For the pre-coalescence systems, contamination from neighboring galaxy was prevented by the use of masks. In all cases, we include the outer low surface brightness regions of the galaxies, until the signal was at the same level as the background.

\section{Results and discussion}

\subsection{Two-dimensional $\mathrm{H} \alpha / \mathrm{H} \beta$-based extinction in pre- and post-coalescence systems}

The two-dimensional $\mathrm{H} \alpha / \mathrm{H} \beta$-based extinction maps of the sample galaxies cover areas of several $\mathrm{kpc}$ on a side, ranging from about $2.5 \mathrm{kpc}$ in highly obscured systems (e.g., IRAS 17208-0014) to 10-12 kpc in galaxy pairs (e.g., IRAS $08572+3915$ or IRAS $14348-1447)$. The angular resolution of the data provides extinction values on scales of between 0.7 and $3 \mathrm{kpc}$, depending on the redshift of the source (see specific values for each galaxy in Table 1). On these scales, the extinction maps (Fig. 1) show that the dust in ULIRGs is not uniformly distributed. It shows instead a patchy structure including almost transparent regions with very low dust content, and other regions that are highly absorbed where, in the optical domain, only a lower limit to the extinction can be derived.

The visual extinction $\left(A_{\mathrm{V}}=3.1 \times E(B-V)\right)$ ranges from $A_{\mathrm{V}} \leq 0.2 \mathrm{mag}$, generally measured in the external regions, to $A_{\mathrm{V}} \simeq 9.0 \mathrm{mag}$, measured in the central kpc of of IRAS 17208 0014. The highest extinctions are usually being measured in the nuclear regions, whose values cover a broad range of $A_{\mathrm{V}}$ between 0.4 and $7.7 \mathrm{mag}, 69 \%$ of the nuclei having $A_{\mathrm{V}}>2.0 \mathrm{mag}$. These high extinction values are similar to those measured in the $\mathrm{Pa} \alpha$ emitting regions of LIRGs, whose average extinctions are found to be $A_{\mathrm{V}}=3-6 \mathrm{mag}$ (Alonso-Herrero et al. 2006) but lower than the values derived for ULIRGs using the nearIR $\mathrm{Pa} \alpha / \mathrm{Br} \gamma$ line ratio, where visual extinctions $\left(A_{\mathrm{V}}\right)$ in excess of 10 magnitudes are relatively common in ULIRGs (Murphy et al. 2001). Both optical and near-IR extinction values are also systematically lower than the Spitzer mid-IR based ones derived for local ULIRGs, which are found to be $<20-30$ mag for PAHemitting normal starbursts, and $\gg 30$ mag for centrally concentrated energy sources with no PAH emission (Imanishi et al. 2007).
Pre- and post-coalescence systems are in different phases of the evolutionary merging process, and therefore their dust and gas content and distribution could reflect this. Pre- and postcoalescence systems do have similar nuclear extinctions with average values of 3.0 and $2.5 \mathrm{mag}$, respectively. However, while extinction values for post-coalescence nuclei tend to be between 2 and 3 mag of visual extinction, the values for pre-coalescence nuclei appear to be more widely distributed over the entire 0.5 mag $\leq A_{\mathrm{V}} \leq 8$ mag range (Fig. 2 center and Table 1 for specific values).

The median extinction derived for entire galaxies (Fig. 2 right) show a clear indication that extinction in the circumnuclear and external regions is substantially lower than in the nuclei. While only $31 \%$ of all nuclei have visual extinctions of 2 mag or less, $60 \%$ of the galaxies do have median extinctions with those low values. Of all the systems, pre-coalescence ULIRGs show on average a difference of 1.2 mag between the median visual extinction for the entire galaxy $(1.8 \mathrm{mag})$ and the nuclear regions (3.0 mag). Post-coalescence systems do however show similar values $(2.1$ and $2.5 \mathrm{mag}$ for median and nuclear extinctions, respectively). This behavior is clearly explained as a consequence of the different morphology of pre- and postcoalescence systems. Pre-coalescence systems contain several low-extinction $\mathrm{H} \alpha$ high-surface brightness regions at distances of several kpc away from the nucleus associated with star formation along the tidal tails and candidates to tidal dwarf galaxies (Monreal-Ibero et al. 2007). Post-coalescence systems are on the other hand more compact and dominated by the emission from the nucleus. The importance of these effects in deriving star formation rates in dust-enshrouded systems is discussed next.

\subsection{Implications of the extinction structure in the derivation of the star formation rates}

Star formation rates $(S F R)$ in galaxies are derived using different tracers, from ultraviolet light and optical forbidden lines ([O II] $3727 \AA$ ), to hydrogen recombination lines (e.g. $\mathrm{H} \alpha, \mathrm{Pa} \alpha, \mathrm{Br} \gamma$ ), and $I R$ broad-band flux (see e.g. Kennicutt 1998). The H $\alpha$ line is the most intense emission line in the optical, and shifts into the near- and mid-IR spectral range for intermediate and high- $z$. galaxies. Because of that, it is one of the most common spectral features used in deriving $S F R$ in all kinds of galaxies at all redshifts.

For obscured and dust-enshrouded galaxies such the low- $z$ ULIRGs and the high- $z$ submillimeter and $24 \mu \mathrm{m}$ bright Spitzer galaxies, the extinction correction applied has important consequences in the reliability of the $\mathrm{H} \alpha$-based SFR. The SFR and $\mathrm{H} \alpha$ luminosity are directly proportional as given by the expression

$\operatorname{SFR}\left(M_{\odot} \mathrm{yr}^{-1}\right)=7.9 \times 10^{-42} L_{\mathrm{H} \alpha}\left(\mathrm{erg} \mathrm{s}^{-1}\right)$

for a continuous star formation, a Salpeter initial mass function with upper and lower mass limits of 100 and $0.1 M_{\odot}$, respectively, and assuming that all potentially ionizing photons ionize the surrounding interstellar medium (Kennicutt 1998). Most dust extinction in low- $z$ ULIRGs to date are obtained through narrow (typically 1 to 1.5 arcsec) long-slit spectra along a given orientation centered on the nucleus of the galaxy (if post-coalescence), or connecting the nuclei of interacting pairs (if pre-coalescence). Observational difficulties arising from differential atmospheric refraction and positioning of the galaxy on the slit translate into an increased uncertainty in the measured values. Since the width of the slit does not cover the entire $\mathrm{H} \alpha$ emitting regions (see Fig. 1 for angular extent and structures), the extinction values derived from these data will be dominated by the emission from the 

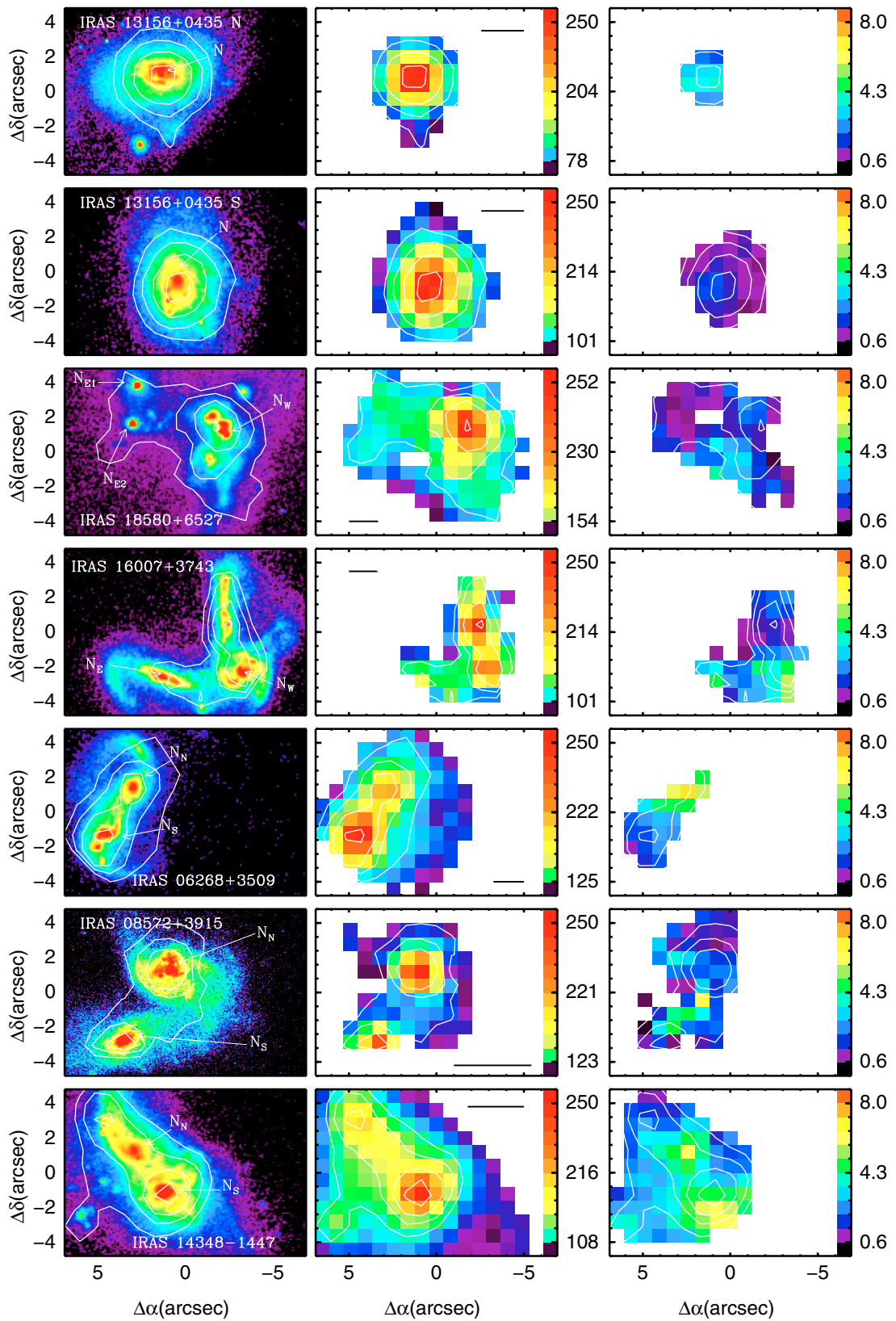

Fig. 1. a. From left to right, HST WFPC2/F814W image, $\mathrm{H} \alpha$ and extinction $A_{\mathrm{V}}$ INTEGRAL maps for a set of ULIRGs. The extinction maps were derived using the $\mathrm{H} \alpha / \mathrm{H} \beta$ line ratio. The HST and $\mathrm{H} \alpha$ maps are represented in logarithmic scale, whereas for the extinction map the scale is linear. As a reference, the white contours shown on every map represent different $\mathrm{H} \alpha$ intensity levels of each galaxy. For the $\mathrm{H} \alpha$ map, we included a color code, which is given in relative flux units. Each pixel of the INTEGRAL maps represents the fiber size of the fiber used (0!' 9$)$. The horizontal scale represents $5 \mathrm{kpc}$. Galaxies orientation is north up, east to the left. nucleus. This would underestimate the $\mathrm{H} \alpha$ luminosity, and therefore the $S F R$, by an average factor of about 4 and 7 in the preand post-coalescence ULIRGs, respectively (see Table 2 for the specific ratios of the observed nuclear to total $\mathrm{H} \alpha$ flux (Col. 3), and the corresponding extinction corrected luminosities (Cols. 5 and 8) for each galaxy.

For the typical median ULIRG extinction $\left(A_{V} \simeq 2.0\right.$ see Fig. 2 right panel), the use of observed $\mathrm{H} \alpha$ luminosities underestimates the $S F R$ by a factor 6 with respect to the extinctioncorrected value. Only $27 \%$ of the galaxies in the sample have de-reddened $S F R$ of less than $20 M_{\odot} \mathrm{yr}^{-1}$, whereas for the uncorrected $S F R$ this percentage increases to $87 \%$. The total dereddened $S F R$ of the present ULIRGs sample ranges from about 10 to $300 M_{\odot} \mathrm{yr}^{-1}$, for a similar range of $S F R$ values for preand post-coalescence systems (see Table 3 ). Finally, we note that because of the lower $\mathrm{S} / \mathrm{N}$ ratio of the $\mathrm{H} \beta$ line, the extinction maps do not cover the same field as the $\mathrm{H} \alpha$ maps (see Fig. 1). This would lead to an additional underestimate of the two-dimensional extinction corrected $\mathrm{H} \alpha$ fluxes (and thus of the $S F R$ values). In any case, and since this mainly affects the outer low-surface brightness regions, no significant changes should be expected.

The $S F R$ is also found to be proportional to the $L_{\mathrm{IR}}$. Considering continuous bursts of 10-100 Myr and applying the same range of IMF parameters as used in Eq. (1) above, the proportionality is given by Kennicutt (1998)

$\operatorname{SFR}\left(M_{\odot} \mathrm{yr}^{-1}\right)=4.5 \times 10^{-44} L_{\mathrm{IR}}\left(\mathrm{erg} \mathrm{s}^{-1}\right)$.

The $S F R$ as derived from the IR luminosity is systematically higher than that obtained from the $\mathrm{H} \alpha$ measurements (see Table 3). The ratio $S F R(I R) / S F R\left(L(H \alpha)_{\text {corr }}\right)$ covers a wide range, from about 2 to 10 , and tends to be smaller in galaxies with higher nuclear extinctions, i.e., IRAS12112+0305, IRAS14348-1447, and IRAS16007+3743. This is because of the large amounts of dust obscuring the nuclear regions, which cause the innermost regions to be more optically thick to optical 

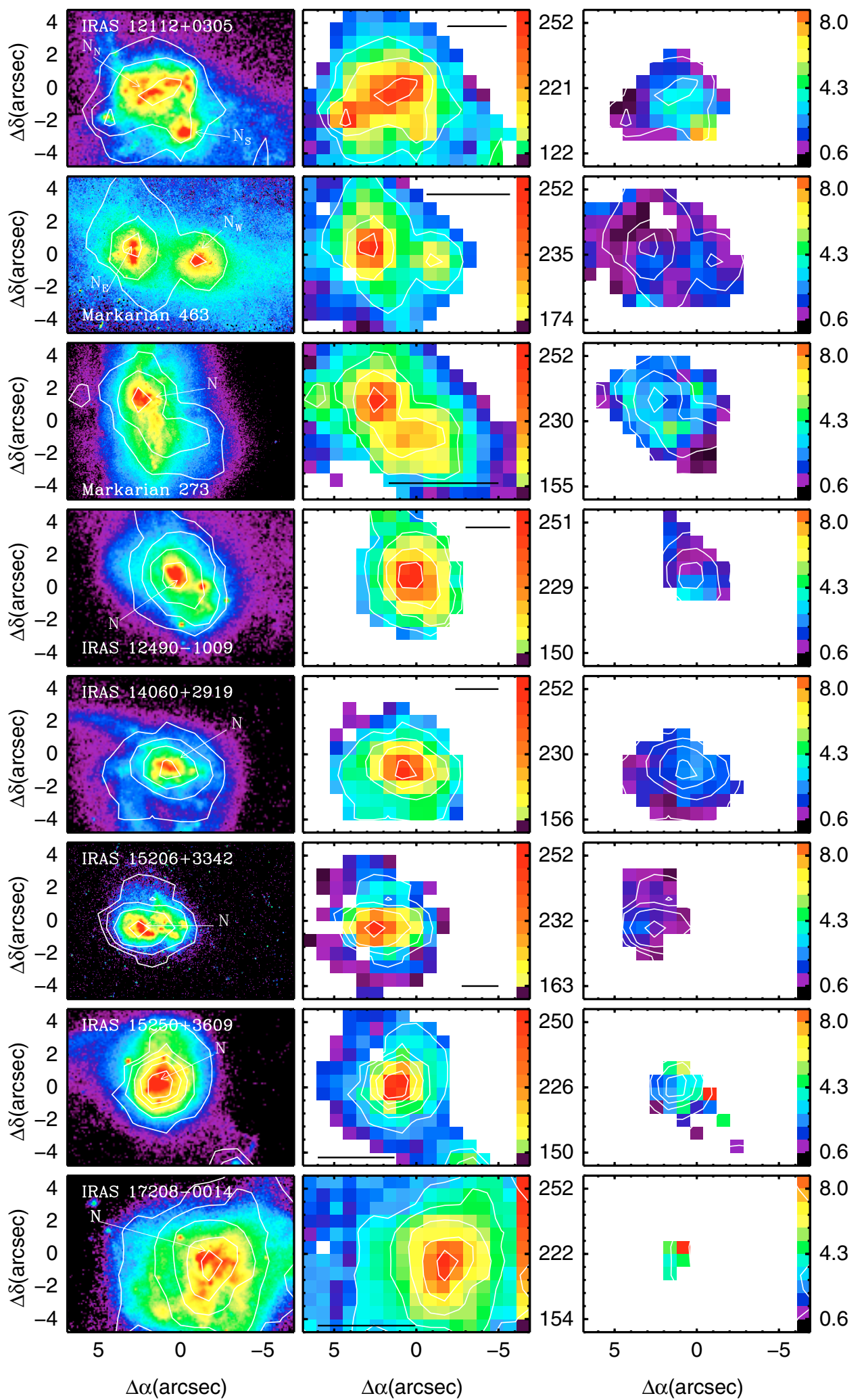

Fig. 1. b. Same as Fig. 1a, but for a different set of ULIRGs.

than far infrared radiation. Thus, even by applying the twodimensional extinction corrections to the observed $\mathrm{H} \alpha$ fluxes, the derived SFRs would represent $10 \%$ to $50 \%$ of the IR-based $S F R$ value, which is assumed to be free of extinction effects, and therefore represent the true SFRs.

\subsection{Implications of the extinction structure for the derivation of the dynamical mass}

Dynamical masses $\left(\propto \sigma^{2} \times R_{\mathrm{hm}}\right.$ ) of galaxies are often derived from measurements of the velocity dispersion of the stars or gas, and assuming the radius $\left(R_{\mathrm{hm}}\right)$ where half of the mass is 

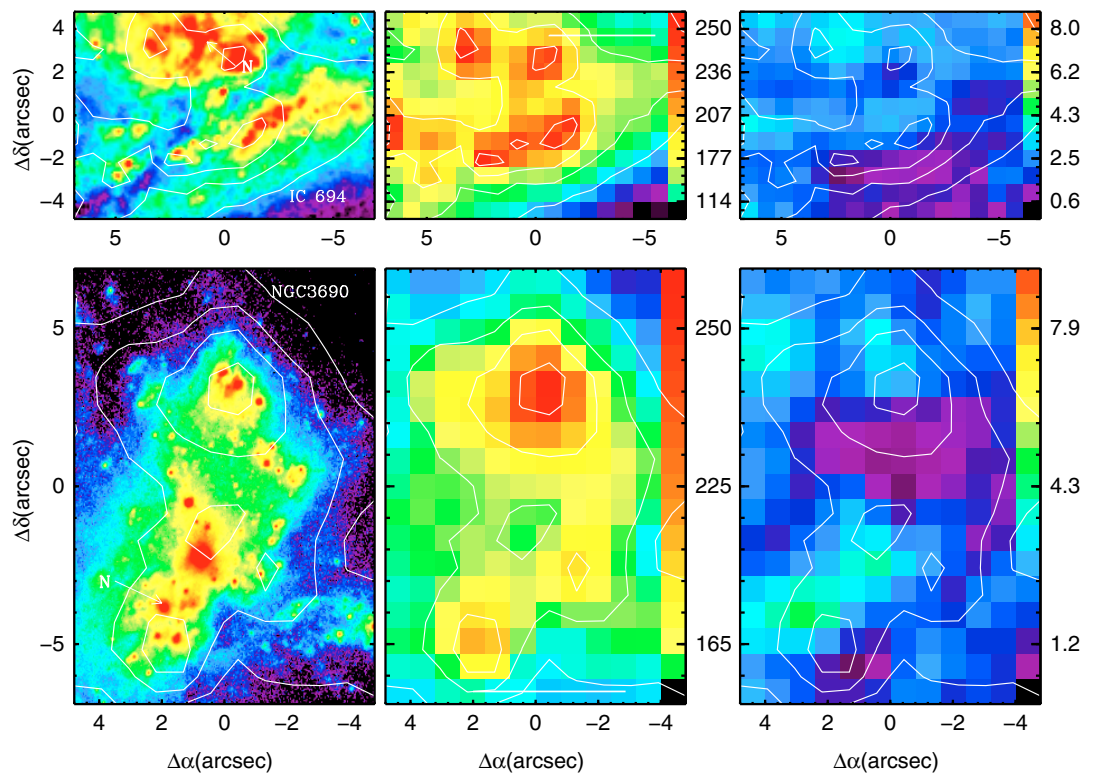

Fig. 1. c. Same as Fig. 1a, but for Arp 299, an interacting galaxy formed by NGC 3690 (West) and IC 694 (East). In this case the scale represents $1 \mathrm{kpc}$.
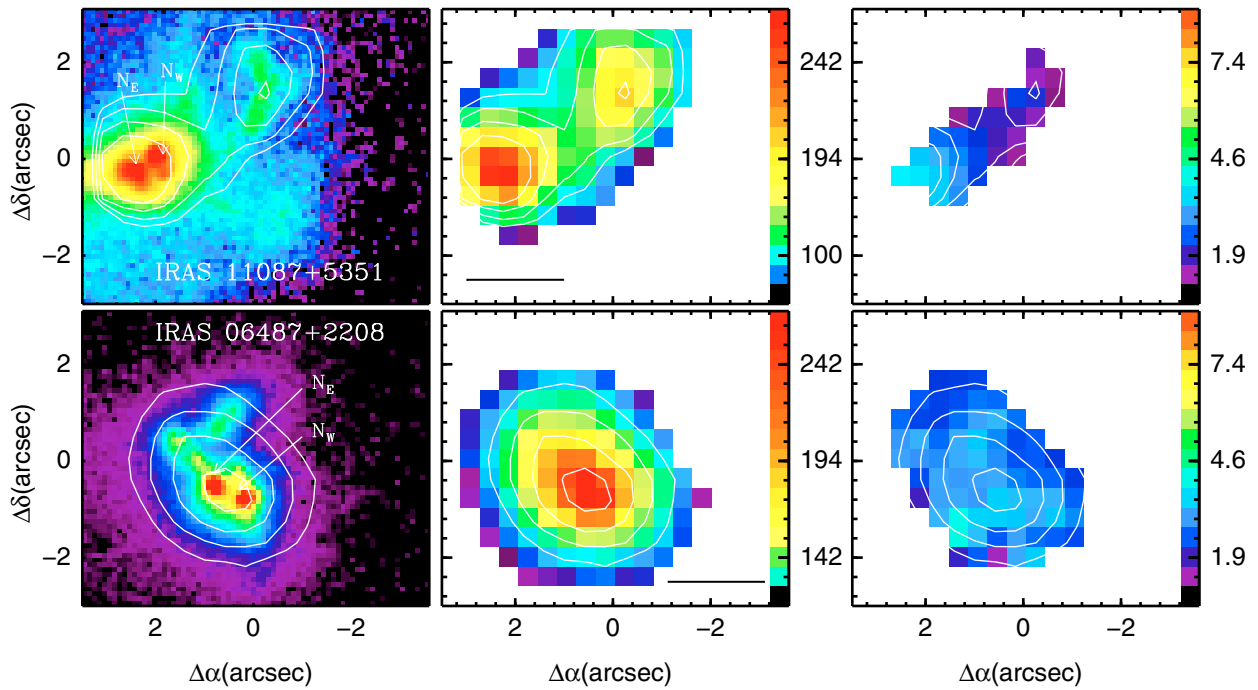

Fig. 1. d. Same as Fig. 1a, but for two different ULIRGs observed with a fiber size of 0.'45.

contained, is known. While the velocity dispersion can be directly obtained, to first order, from the full-width-half-maximum of the absorption/emission line profiles, the half-mass radius is not a direct observable and has to be inferred from the half-light radius.

The most common lines used to derive velocity dispersions in low- $z$ ULIRGs and their high- $z$ analogs are in the optical (e.g., $\mathrm{H} \beta$ and $\mathrm{H} \alpha$ ) and near-IR (e.g., Br $\gamma$, CO stellar absorption bands) rest-frame, and are therefore affected by non-uniform extinction (see Sect. 5.1). The behavior of the extinction in ULIRGs with large values in the nuclear regions and an outward decreasing gradient in the circumnuclear and extranuclear regions (see Sect. 5.1 and Fig. 1), produces an apparent stellar light distribution that differs from that of the true mass distribution. On the one hand, for a given rest-frame wavelength, the half-light radius will always be an upper limit to the true $R_{\mathrm{hm}}$ as the light from stars in the circum- and extranuclear regions are less obscured than those in the nuclear regions. On the other hand, the extinction has a strong wavelength dependence becoming higher towards bluer wavelengths. For a given galaxy, the halflight radius derived in the optical will always be systematically larger than those derived in the near-IR. This effect is observed in our sample galaxies where for those galaxies with available $H S T$ optical $(0.8 \mu \mathrm{m}$, WFPC2 F814W) and near-IR $(1.6 \mu \mathrm{m}$, NICMOS2 F160W) images (see Table 1), the optical half-light radius is on average 2.2 times the corresponding value in the near-IR, in some systems up to 5 times larger. Therefore, in obscured systems such as local and high- $z$ ULIRGs, the use of optical (red) rest-frame light will produce an average overestimate of the dynamical mass by a factor of 2.2 merely because of extinction effects. This factor could be even larger if effective radii are measured in the blue (B) or visual (V) rest-frames (see specific values in Table 1), as frequently performed for in high- $z$. galaxies.

In addition to these extinction effects, other effects such as stellar population gradients can also be important. Since young 
Table 2. Observed $\mathrm{H} \alpha$ fluxes and extinction corrected luminosities for the sample galaxies.

\begin{tabular}{|c|c|c|c|c|c|c|c|}
\hline Galaxy & $F(\mathrm{H} \alpha)_{\mathrm{obs}}{ }^{a}$ & $F(\mathrm{H} \alpha)_{\text {nuclear } / \text { total }^{b}}$ & $L(\mathrm{H} \alpha)_{\mathrm{obs}}{ }^{c}$ & $L(\mathrm{H} \alpha)_{\text {extcorr }}^{d}$ & $L(\mathrm{H} \alpha)_{\operatorname{med}-\mathrm{ext}}^{e}$ & $L_{\mathrm{nuc}}(\mathrm{H} \alpha)_{\mathrm{obs}}{ }^{f}$ & $L_{\mathrm{nuc}}(\mathrm{H} \alpha)_{\mathrm{corr}^{g}}^{g}$ \\
\hline IRAS $13156+0435 \mathrm{~N}$ & 0.9 & 0.12 & 3.0 & 21.0 & 27.1 & 0.36 & 4.6 \\
\hline IRAS $13156+0435 \mathrm{~S}$ & 1.2 & 0.09 & 3.9 & 9.0 & 6.1 & 0.35 & 1.3 \\
\hline \multirow[t]{2}{*}{ IRAS $18580+6527$} & 2.5 & [E] 0.01 & 21.6 & 78.0 & 68.2 & 0.22 & 0.4 \\
\hline & & [W] 0.28 & & & & 6.05 & 15.9 \\
\hline \multirow{2}{*}{ IRAS $16007+3743$} & 2.6 & [E] 0.03 & 25.1 & 152.0 & 134.7 & 0.75 & 17.7 \\
\hline & & [W] 0.22 & & & & 5.52 & 97.0 \\
\hline \multirow{2}{*}{ IRAS 06268+3509 } & 0.6 & [N] 0.09 & 4.7 & 121.0 & 28.0 & 0.42 & 66.5 \\
\hline & & [S] 0.18 & & & & 0.85 & 4.4 \\
\hline \multirow[t]{2}{*}{ IRAS $08572+3915$} & 5.8 & {$[\mathrm{~N}] 0.08$} & 4.6 & 15.5 & 21.1 & 0.37 & 2.0 \\
\hline & & [S] 0.05 & & & & 0.23 & 0.3 \\
\hline \multirow[t]{2}{*}{ IRAS $14348-1447$} & 15.0 & {$[\mathrm{~N}] 0.03$} & 25.6 & 381.0 & 333.0 & 0.77 & 52.3 \\
\hline & & [S] 0.08 & & & & 2.05 & 31.5 \\
\hline Arp 299/NGC 3690 & 181.0 & $0.03^{h}$ & 4.0 & 29.0 & 21.5 & 0.12 & 0.9 \\
\hline Arp 299/IC 694 & 89.0 & $0.03^{h}$ & 2.0 & 16.0 & 11.9 & 0.06 & 6.2 \\
\hline \multirow{2}{*}{ IRAS $12112+0305$} & 5.8 & {$[\mathrm{~N}] 0.08$} & 7.6 & 213.0 & 34.0 & 0.60 & 9.0 \\
\hline & & [S] 0.02 & & & & 0.15 & 155.0 \\
\hline \multirow{2}{*}{ Mrk 463} & 36.9 & [E] 0.25 & 21.8 & 56.0 & 48.2 & 5.45 & 10.4 \\
\hline & & [W] 0.02 & & & & 0.44 & 1.8 \\
\hline IRAS 06487+2208 & 2.5 & 0.19 & 13.9 & 117.0 & 96.3 & 2.64 & 23.3 \\
\hline IRAS $11087+5351$ & 0.8 & 0.12 & 4.4 & 11.0 & 12.0 & 0.53 & 7.4 \\
\hline $\operatorname{Mrk} 273^{i}$ & - & 0.08 & - & - & - & - & - \\
\hline IRAS 17208-0014 & 17.9 & 0.10 & 7.7 & 160.2 & $-^{j}$ & 0.7 & 43.0 \\
\hline IRAS $15250+3609$ & 4.4 & 0.26 & 3.2 & 87.5 & 25.0 & 0.83 & 4.4 \\
\hline IRAS 12490-1009 & 1.0 & 0.13 & 2.6 & 12.0 & 8.1 & 0.34 & 0.9 \\
\hline IRAS $14060+2919$ & 2.3 & 0.18 & 8.2 & 45.0 & 30.8 & 1.48 & 10.7 \\
\hline IRAS $15206+3342$ & 42.7 & 0.38 & 171.8 & 322.0 & 348.7 & 65.3 & 158.5 \\
\hline
\end{tabular}

${ }^{a}$ Integrated $\mathrm{H} \alpha$ observed flux, with units $\times 10^{-14} \mathrm{erg} \mathrm{s}^{-1} \mathrm{~cm}^{-2}$. Note that in the cases of IRAS $13156+0435$ and Arp 299 we derived the integrated flux for each galaxy of the interacting pair using their individual pointings; ${ }^{b}$ ratio between the $\mathrm{H} \alpha$ nuclear, identified as the peak position in the optical continuum and total observed fluxes; ${ }^{c}$ observed $\mathrm{H} \alpha$ luminosity derived from the integrated flux in Col. 2 . Units in $\times 10^{41}$ erg s${ }^{-1} ;{ }^{d}$ integrated two-dimensional extinction corrected $\mathrm{H} \alpha$ luminosity, with units in $\times 10^{41} \mathrm{erg} \mathrm{s}^{-1}$; ${ }^{e}$ integrated $\mathrm{H} \alpha$ extinction-corrected luminosity, derived using the total observed flux and the median extinction, with units in $\times 10^{41} \mathrm{erg} \mathrm{s}^{-1} ;{ }^{f}$ observed nuclear H $\alpha$ luminosity with units in $\times 10^{41} \mathrm{erg} \mathrm{s}^{-1} ;{ }^{g}$ extinction corrected nuclear $\mathrm{H} \alpha$ luminosity, derived using the nuclear extinction of each individual galaxy. Units in $\times 10^{41} \mathrm{erg} \mathrm{s}^{-1} ;{ }^{h}$ for this particular galaxy we have calculated the ratio using the position of the true nucleus of the galaxy, which does not coincide with the optical one (García-Marín et al. 2006); ${ }^{i}$ the data for Mrk 273 do not have an absolute flux calibration. Hence, only the relative measurement between the nuclear and integrated $\mathrm{H} \alpha$ flux is given here; ${ }^{j}$ as shown in Fig. 1b, the area of this galaxy with available extinction values is limited, also presenting a very large peak value. The use of this value for correcting the integrated flux is not valid and therefore is not included in the table.

Table 3. $\mathrm{H} \alpha$ - and IR-based star formation rates for the sample of ULIRGs.

\begin{tabular}{lccccc}
\hline \hline Galaxy & $\log \left(L_{\mathrm{IR}}\right)^{a}$ & $S F R\left(L(\mathrm{H} \alpha)_{\mathrm{obs}}\right)^{b}$ & $S F R\left(L(\mathrm{H} \alpha)_{\text {ed-ext }^{c}}\right)$ & $S F R\left(L(\mathrm{H} \alpha)_{\mathrm{corr}}\right)^{d}$ & $S F R(I R)^{e}$ \\
\hline IRAS 13156+0435 & 12.13 & 5.5 & 26.2 & 23.7 & 232.9 \\
IRAS 18580+6527f & 12.26 & 17.1 & 53.8 & 61.6 & 318.7 \\
IRAS 16007+3743 & 12.11 & 19.8 & 106.4 & 120. & 222.5 \\
IRAS 06268+3509 & 12.51 & 3.7 & 22.1 & 95.6 & 493.5 \\
IRAS 08572+3915 & 12.17 & 3.6 & 16.7 & 12.2 & 255.5 \\
IRAS 14348-1447 & 12.39 & 20.2 & 263.1 & 301.0 & 424.1 \\
Arp 299 & 11.81 & 4.7 & 26.3 & 35.3 & 111.5 \\
IRAS 12112+0305 & 12.37 & 6.0 & 26.9 & 168.3 & 405.0 \\
Mrk 463 & 11.81 & 17.2 & 38.0 & 44.2 & 111.5 \\
IRAS 06487+2208 & 12.57 & 11.0 & 76.0 & 92.4 & 641.8 \\
IRAS 11087+5351 & 12.13 & 3.5 & 9.5 & 8.7 & 233.0 \\
Mrk 273 & 12.18 & - & - & - & 216.5 \\
IRAS 17208-0014 & 12.43 & 6.1 & - & 126.5 & 465.0 \\
IRAS 15250+3609 & 12.09 & 2.5 & 19.7 & 69.1 & 212.5 \\
IRAS 12490-1009 & 12.07 & 2.0 & 6.4 & 9.5 & 203.0 \\
IRAS 14060+2919 & 12.18 & 6.4 & 24.3 & 35.5 & 261.5 \\
IRAS 15206+3342 & 12.27 & 135.7 & 275.5 & 254.3 & 321.6 \\
\hline
\end{tabular}

${ }^{a}$ IR luminosity derived following Sanders \& Mirabel (1996); ${ }^{b}$ SFR derived using the total observed $\mathrm{H} \alpha$ flux given in Col. 2 of Table 2. Units are $M_{\odot} \mathrm{yr}^{-1} ;{ }^{c}$ SFR derived using the $\mathrm{H} \alpha$ median extinction corrected values as specified in Table 2 Col. 6 . Units are $M_{\odot} \mathrm{yr}^{-1} ;{ }^{d} \mathrm{SFR}$ calculated using the two-dimensional extinction corrected $\mathrm{H} \alpha$ luminosity as given in Table 2 Col. 5. Units are $M_{\odot} \mathrm{yr}^{-1} ;{ }^{e} \mathrm{SFR}$ based on the IR-based luminosity values as given in Col. 2 of the present table. Units are $M_{\odot} \mathrm{yr}^{-1} ;{ }^{f}$ strong evidence for a Seyfert nucleus, for which we have not corrected this values. 

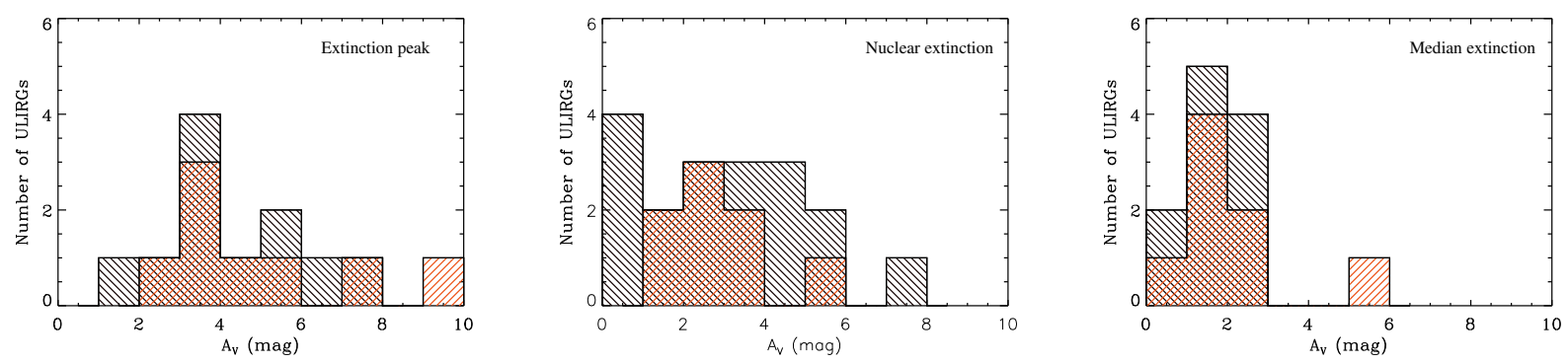

Fig. 2. Distribution of the extinction values in the sample of ULIRGs. The black dashed histogram indicates pre-coalescence systems. The red dashed histogram indicates post-coalescence galaxies. Left panel: distribution of the sample of galaxies according to the peak of extinction for each ULIRG. Center panel: galaxy distribution according to the extinction in the nuclear regions. Right panel: distribution of the sample of galaxies according to the median extinction of the systems. Note that in the cases of IRAS 13156+0435 and Arp 299, we derived the median for each galaxy of the interacting system using the individual pointings with the SB2 bundle.

(10 Myr or less) massive stars and older (100 Myr to Gyr) stellar populations have mass-to-light ratios that differ by up to two orders of magnitude in the optical and near-IR (e.g. Starburst99, Leitherer et al. 1999), their non-uniform spatial distribution is also relevant to evaluating the uncertainty in the measured halflight radius, and therefore in the dynamical mass. With only two filters available for most of the sample galaxies, a detailed analysis of these effects is beyond the scope of the present work.

\section{Summary}

This paper has presented the study of the two-dimensional extinction structure of a representative sample of 17 ULIRGs, 9 pre-coalescence (i.e., interacting pairs with projected nuclear separations of at least $1.5 \mathrm{kpc}$ ), and 8 post-coalescence (either single nucleus or double nuclei with projected separations of less than $1.5 \mathrm{kpc}$ ). The extinction maps are based on the measurement of the $\mathrm{H} \alpha / \mathrm{H} \beta$ line ratio obtained from integral field spectroscopy with the instrument INTEGRAL on the William Herschel telescope. In comparison with more classical narrow long-slit spectroscopy, this study infers the two-dimensional extinction structure of ULIRGs on kpc scales over areas of a few to several kpc on a side. The main results are summarized below:

- In agreement with previous studies, the analysis of our data has detected a very complex and patchy extinction structure in ULIRGs on scales of $\mathrm{kpc}$, from basically transparent regions to others so deeply embedded in dust that only a lower limit to their extinction can be derived $\left(A_{\mathrm{V}} \simeq 0.0 \mathrm{mag}\right.$ to $A_{\mathrm{V}} \simeq 9.0 \mathrm{mag}$ ). Nuclear extinction values of the present galaxy sample cover a broad range in $A_{V}$ from about 0.5 to $8 \mathrm{mag}, 69 \%$ of the nuclei having $A_{V}>2.0 \mathrm{mag}$. Extinction in the external regions is substantially lower than in the nuclei with $60 \%$ of the ULIRGs in the sample having a median $\mathrm{A}_{V}$ for the entire galaxy of less than two magnitudes.

- ULIRGs classified as pre- and post-coalescence exhibit significant differences in their extinction properties. While postcoalescence nuclei tend to cluster around $A_{\mathrm{V}}$ values of 2 to $3 \mathrm{mag}$, pre-coalescence nuclei appear more homogeneously distributed over the entire $0.4 \mathrm{mag} \leq A_{\mathrm{V}} \leq 7.7 \mathrm{mag}$ range. Pre-coalescence ULIRGs show on average a difference of $1.2 \mathrm{mag}$ between the nuclear $\left(A_{\mathrm{V}}\right.$ of $\left.3.0 \mathrm{mag}\right)$ and median extinction for the entire system ( $A_{\mathrm{V}}$ of $\left.1.8 \mathrm{mag}\right)$, while post-coalescence systems do show similar values $(2.1$ and 2.5 mag for median and nuclear extinctions, respectively). This behavior is explained as a consequence of the different distribution of the star-forming regions in pre- and post-coalescence systems. Pre-coalescence systems contain several low-extinction $\mathrm{H} \alpha$ high-surface brightness regions at distances of several kpc away from the nucleus associated with star formation along the tidal tails. Post-coalescence systems are more compact and dominated by the emission from the nuclear regions.

- Considering the median extinctions measured in the sample galaxies $\left(A_{\mathrm{V}} \simeq 2.0\right)$, the $S F R$ values based on the de-reddened $\mathrm{H} \alpha$ fluxes increase by a factor 6 with respect to those derived from the observed $\mathrm{H} \alpha$ fluxes. The $\mathrm{H} \alpha$-based extinctioncorrected $S F R$ ranges from about 10 to $300 M_{\odot} \mathrm{yr}^{-1}$. The dereddened $S F R$ is less than $20 M_{\odot} \mathrm{yr}^{-1}$ in $27 \%$ of the galaxies, whereas for the observed $S F R$ this percentage increases up to $87 \%$. No significant differences in the $S F R$ have been found between the pre- and post-coalescence systems.

- In all galaxies, the IR-based $S F R$ is higher than the dereddened $\mathrm{H} \alpha$ value. The ratio ranges from 2 to 10 , with the smaller differences in galaxies with higher extinction in their nuclear regions. Thus, assuming IR luminosities measure the true $S F R$ in these systems, SFRs based on full twodimensional extinction corrected $\mathrm{H} \alpha$ luminosities recover between $10 \%$ and $50 \%$ of the true SFR.

- Because of the high nuclear extinctions and the outward decreasing extinction gradient, the optical ( $I$-band) half-light radius in the sample of ULIRGs is on average a factor 2.2 larger than the corresponding value in the near-IR ( $H$-band), and in some systems even a factor 5 larger. Dynamical masses in ULIRGs and their high $-z$ analogs would be on average overestimated by a factor 2.2 due merely to extinction effects if the effective radius of the galaxy is derived directly from observed rest-frame optical images. This factor could be even larger if bluer broad-band filters (i.e., $V$ and $B$ bands) were used to measure the effective radius as often the case with high- $z$ galaxies. Additional stellar population effects could also be relevant because of their different massto-light ratios, as should be investigated by future detailed studies.

Acknowledgements. This paper uses the plotting package jmaplot, developed by Jesús Maíz-Apellániz. http://dae45. \penalty-\@Miaa.csic.es:

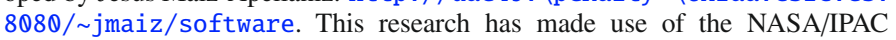
Extragalactic Database (NED) which is operated by the Jet Propulsion Laboratory, California Institute of Technology, under contract with the National Aeronautics and Space Administration.

This work has been supported by the Spanish Ministry of Education and Science, under grant BES-2003-0852, project AYA2002-01055. MG-M is supported by the German federal department for education and research (BMBF) under the project numbers: $50 \mathrm{OS} 0502 \& 50 \mathrm{OS} 0801$. 


\section{References}

Alonso-Herrero, A., Rieke, G. H., Rieke, M. J., et al. 2006, ApJ, 650, 835

Alonso-Herrero, A., García-Marín, M., Monreal-Ibero, A., et al. 2009, [arXiv:0907.5105]

Arribas, S., \& Colina, L. 2003, ASP Conf. Proc., 297, 24

Arribas, S., Mediavilla, E., García-Lorenzo, B., et al. 1997, ApJ, 490, 227

Arribas, S., Carter, D., Cavaller, L., et al. 1998, Proc. SPIE, 3355, 821

Arribas, S., Colina, L., Monreal-Ibero, A., et al. 2008, A\&A, 479, 687

Bedregal, A. G., Colina, L., Alonso-Herrero, A., et al. S. 2009, ApJ, 698, 1852

Bingham, R. G., Gellatly, D. W., Jenkins, C. R., et al. 1994, Proc. SPIE, 2198, 56

Bushouse, H. A., Borne, K. D., Colina, L., et al. 2002, ApJS, 138, 1

Colina, L., Arribas, S., \& Borne, K. D. 1999, ApJ, 527, L13

Colina, L., Arribas, S., Borne, K. D. et al. 2000, ApJ, 533, L9

Colina, L., Borne, K., Bushouse, H., et al. 2001, ApJ, 563, 546

Colina, L., Arribas, S., \& Monreal-Ibero, A. 2005, ApJ, 621, 725

Farrah, D., Rowan-Robinson, M., Oliver, S., et al. 2001, MNRAS, 326, 1333

García-Marín , M., Colina, L., Arribas, S., et al. 2006, ApJ, 650, 850

García-Marín , M., Colina, L., Arribas, S., \& Monreal-Ibero, A. 2009, A\&A, 505, 1319

Genzel, R., Tacconi, L. J., Rigopoulou, D., Lutz, D., \& Tecza, M. 2001, ApJ, 563,527

Howarth, I. D., \& Murray, J. 1988, DIPSO A Friendly Spectrum Analysis Program (Starlink User Note 50; Chilton: Rutherford Appleton Lab.)

Imanishi, M., Dudley, C. C., Maiolino, R., et al. 2007, ApJS, 171, 72
Kennicutt, R. C., Jr. 1998, ARA\&A, 36, 189

Leitherer, C., Schaerer, D., Goldader, J. D., et al. 1999, ApJS, 123, 3

LeFevre, O. et al. 2003, SPIE, 4841, 1670

Lonsdale, C., Farrah, D., \& Smith, H. 2006, Astrophysics Update 2, ed. John W. Mason (Verlag, Heidelberg, Germany: Springer), 285

Low, J., Kleinmann, \& D. E. 1968, AJ, 73, 868

Monreal-Ibero, A., Colina, L., Arribas, S., \& García-Marín, M. 2007, A\&A, 472, 421

Murphy, T. W., Jr., Soifer, B. T., Matthews, K., Armus, L., \& Kiger, J. R. 2001, AJ, 121, 97

Naab, T., Jesseit, R., \& Burkert, A. 2006, MNRAS, 372, 839

Nardini, E., Risaliti, G., Salvati, M., et al. 2008, MNRAS, 385, L130

Origlia, L., \& Leitherer, C. 2000, AJ, 119, 2018

Rieke, G. H., \& Low, F. J. 1972, ApJ, 176L, 95

Risaliti, G., Maiolino, R., Marconi, A., et al. 2006, MNRAS, 365, 303

Roth, M., Kelz, A., Fechner, Th., et al. 2005, PASP, 117, 620

Sanders, D. B., \& Mirabel, I. F. 1996, ARA\&A, 34, 749

Savage, B. D., \& Mathis, J. S. 1979, ARA\&A, 17, 73

Scoville, N. Z., Evans, A. S., Thompson, R., et al. 2000, AJ, 119, 991

Soifer, B. T., Rowan-Robinson, M., Houck, J. R., et al. 1984, ApJ, 278, L71

Surace, J. A., Sanders, D. B., Vacca, W. D., Veilleux, S., \& Mazzarella, J. M. 1998, ApJ, 492, 116

Tacconi, L. J., Genzel, R., Lutz, D., et al. 2002, ApJ, 580, 73

Veilleux, S., Kim, D. C., Sanders, D. B., Mazzarella, J. M., \& Soifer, B. T. 1995, ApJS, 98, 171 